\title{
A REMARK ON A STABILITY THEOREM OF M. MARACHKOFF
}

\author{
JOHN R. HADDOCK
}

Abstract. By placing certain conditions on $f(t, x)$ for the system of ordinary differential equations

$$
x^{\prime}=f(t, x), \quad f(t, 0) \equiv 0,
$$

Marachkoff weakened the conditions on the Liapunov function of the classical asymptotic stability theorem of Liapunov theory and obtained asymptotic stability of the zero solution of (1). Later, Massera gave a shorter proof of Marachkoff's result. In this note we show that Marachkoff's theorem can be proven without the use of one of the conditions.

1. Introduction. Let $G$ be an open subset of $R^{n}$ containing the origin and consider the system of ordinary differential equations

$$
x^{\prime}=f(t, x) \quad\left({ }^{\prime}=d / d t\right),
$$

where $f:[0, \infty) \times G \rightarrow R^{n}, f(t, 0) \equiv 0$, and (1) is of Carathéodory type $\left[3\right.$, p. 43]. Thus (1) possesses the zero solution and for each $\left(t_{0}, x_{0}\right)$ in $[0, \infty) \times G$ there exists at least one solution $x\left(t, t_{0}, x_{0}\right)$ defined on some $t$ interval containing $t_{0}$.

For a set $B$, we shall denote by $B^{c}$ the complement of $B$. Also, for $\varepsilon>0$, we define $S(\varepsilon)=\{x:|x|<\varepsilon\}$ and $H(\varepsilon)=\{x: \varepsilon / 2 \leqq|x| \leqq \varepsilon\}$, where $|\cdot|$ is any suitable norm in $R^{n}$.

The purpose of this note is to improve conditions on Liapunov functions which insure asymptotic stability of the zero solution of (1). For definitions of terms and symbols, we refer the reader to [2] and Chapter 1 of [4]. To avoid confusion we mention that "infinitesimal upper bound" and "decrescent" are used interchangeably in the literature.

The following is the classical asymptotic stability theorem in Liapunov theory.

Received by the editors September 28, 1970 and, in revised form, April 16, 1971. AMS 1969 subject classifications. Primary 3451.

Key words and phrases. Zero solution, stability, asymptotic stability, Liapunov function, positive definite, negative definite, decrescent, total derivative. 
THEOREM 1 ([4, THEOREM II.4.2]). Suppose there exists a differentiable function $V:[0, \infty) \times G \rightarrow[0, \infty)$ which satisfies the following conditions:

(i) $V(t, 0) \equiv 0$,

(ii) $V$ is positive definite and decrescent, and

(iii) the total derivative

is negative definite (where

$$
V^{\prime}(t, x)=\frac{\partial V(t, x)}{\partial t}+\sum_{i=1}^{n} \frac{\partial V(t, x)}{\partial x_{i}} f_{i}(t, x)
$$

$$
\left.x=\operatorname{col}\left(x_{1}, \cdots, x_{n}\right) \text { and } f(t, x)=\operatorname{col}\left(f_{1}, \cdots, f_{n}\right)\right) .
$$

Then the zero solution of $(1)$ is asymptotically stable.

Observing that the conditions of Theorem 1 are sufficient to insure uniform asymptotic stability of the zero solution of (1), Marachkoff [5] (see also Antosiewicz [1, p. 149] and Massera [6, p. 707]) obtained the following result which does not necessarily imply uniform asymptotic stability.

THEOREM 2 ([5, THEOREM 4]). Suppose, for $f(t, x)$ continuous, there exists $A>0$ and $M>0$ such that $|f(t, x)| \leqq M$ for all $(t, x)$ in $[0, \infty) \times$ $S(A)$. Further, suppose there exists a differentiable function $V:[0, \infty) \times$ $G \rightarrow[0, \infty)$ which satisfies the following conditions.

(i) $V(t, 0) \equiv 0$,

(ii) $V$ is positive definite, and

(iii) the total derivative $V^{\prime}$ for (1) is negative definite.

Then the zero solution of (1) is asymptotically stable.

Although one need only assume that $V$ is absolutely continuous in $t$ uniformly at any point $\left(t_{0}, x_{0}\right)$ and that $V$ is locally Lipschitzian in $x$ (cf. [7, pp. 10-11]), we ask that $V$ be differentiable.

We note that Theorem 2 is not an improvement of Theorem 1 in the strictest sense of the word since, in Theorem 2, it is asked that $f(t, x)$ be bounded on $[0, \infty) \times S(A)$.

2. The results. In Theorem 3 we show that the conclusion of Theorem 2 holds with condition (ii) replaced by a weaker hypothesis. We note that Theorem 3 is primarily a stability result since we are not using a "definiteness" property of the scalar function $V$. We shall rely on the following simple lemma.

Lemma. Suppose there exists $A>0$ and $M>0$ such that $|f(t, x)| \leqq M$ for all $(t, x)$ in $[0, \infty) \times S(A)$. Then, for each $\varepsilon>0(\varepsilon<A)$ there exists $T(\varepsilon)>0$ such that, if there exists $t_{2}, t_{1} \geqq 0$ such that $\left|x\left(t_{2}\right)-x\left(t_{1}\right)\right| \geqq \varepsilon / 2$ and $x(t)$ is in $S(A)$ for all $t_{1} \leqq t \leqq t_{2}$ and some solution $x(t)$ of $(1)$, then $t_{2}-t_{1} \geqq T(\varepsilon)$. 
Proof. Let $\varepsilon>0$ be given with $\varepsilon<A$ and suppose the conditions of the Lemma are satisfied for some solution $x(t)$ of (1). Then

$$
\varepsilon / 2 \leqq\left|x\left(t_{2}\right)-x\left(t_{1}\right)\right| \leqq \int_{t_{1}}^{t_{2}}|f(t, x(t))| d t \leqq M\left(t_{2}-t_{1}\right) .
$$

Let $T(\varepsilon)=\varepsilon / 2 M$. Then $t_{2}-t_{1} \geqq T(\varepsilon)$ and the proof is complete.

THEOREM 3. Suppose there exists $A>0$ and $M>0$ such that $|f(t, x)| \leqq M$ for all $(t, x)$ in $[0, \infty) \times S(A)$ and suppose there exists $a$ differentiable function $V:[0, \infty) \times G \rightarrow[0, \infty)$ which satisfies the following conditions:

(i) $V(t, 0) \equiv 0$,

(ii) $V(t, x) \geqq 0$ for all $(t, x)$ in $[0, \infty) \times S(A)$, and

(iii) the total derivative $V^{\prime}$ for (1) is negative definite.

Then the zero solution of (1) is asymptotically stable.

Proof. Let $t_{0} \geqq 0$ and $\varepsilon>0$ be given with $\varepsilon<A$ and let $T(\varepsilon)$ of the Lemma be chosen. Without loss of generality we may assume $V^{\prime}(t, x)$ is negative definite on $S(A)$ and hence, there exists $\alpha=\alpha(\varepsilon)>0$ such that $V^{\prime}(t, x) \leqq-\alpha$ for all $(t, x)$ in $[0, \infty) \times H(\varepsilon)$. Since $V$ is continuous and $V\left(t_{0}, 0\right)=0$, there exists $\delta>0$ such that $V\left(t_{0}, x_{0}\right)<\alpha T(\varepsilon)$ whenever $\left|x_{0}\right|<\delta<\varepsilon / 2$. We show that the zero solution is stable past $t_{0}$. Suppose there exists $\left|x_{0}\right|<\delta$ and $t_{2} \geqq t_{1}>t_{0}$ and some solution $x\left(t, t_{0}, x_{0}\right)$ of (1) such that $\left|x\left(t_{1}, t_{0}, x_{0}\right)\right|=\varepsilon / 2,\left|x\left(t_{2}, t_{0}, x_{0}\right)\right|=\varepsilon$ and $x\left(t, t_{0}, x_{0}\right)$ is in $H(\varepsilon)$ whenever $t_{1} \leqq t \leqq t_{2}$. Integrating $V^{\prime}$ along $x\left(t, t_{0}, x_{0}\right)=x(t)$ we obtain

$$
\begin{aligned}
V\left(t_{2}, x\left(t_{2}\right)\right) & \leqq V\left(t_{1}, x\left(t_{1}\right)\right)-\alpha\left(t_{2}-t_{1}\right) \\
& \leqq V\left(t_{0}, x_{0}\right)-\alpha\left(t_{2}-t_{1}\right)<\alpha T(\varepsilon)-\alpha T(\varepsilon)=0 .
\end{aligned}
$$

This contradicts $V \geqq 0$ and hence, since $t_{0}$ was arbitrary, the zero solution is stable.

The remainder of the proof follows from the proof of Theorem 2 which was given by Massera and is therefore omitted.

We shall now prove that the conditions of Theorem 3 actually imply that $V$ is positive definite. Since the proof of this result does not rely on the conclusion of Theorem 3 , it is immediately clear that the above proof could have been entirely omitted. This proof has been retained for informative purposes since a technique was developed for obtaining a stability result without having to use the positive definiteness property of $V$.

THEOREM 4. Under the conditions of Theorem 3, $V$ is positive definite. 
Proof. Suppose not. Then there exists $\varepsilon>0$ such that, for each $\delta>0$ there exists $|x|=\varepsilon$ and $t \geqq 0$ such that $V(t, x)<\delta$. Clearly, we may assume $3 \varepsilon / 2<A$. Since $V^{\prime}$ is negative definite (on $S(A)$ ), there exists $\alpha>0$ such that $V^{\prime}(t, x) \leqq-\alpha$ for all $t \geqq 0$ and all $\varepsilon / 2 \leqq|x| \leqq 3 \varepsilon / 2$. Let $T(\varepsilon)$ of the Lemma be chosen and let $\delta_{0}>0$ be chosen sufficiently small such that $\delta_{0}<\alpha T(\varepsilon)$. Then there exists $\left|x_{0}\right|=\varepsilon$ and $t_{0} \geqq 0$ such that $V\left(t_{0}, x_{0}\right)<\delta_{0}$. Consider the solution $x\left(t, t_{0}, x_{0}\right)=x(t)$. Suppose $\varepsilon / 2<|x(t)|<3 \varepsilon / 2$ for all $t \geqq t_{0}$. Since $V^{\prime}$ is negative definite, we can integrate $V^{\prime}$ along $x(t)$ to contradict $V$ bounded from below. Hence there exists $t_{2}>t_{1} \geqq t_{0}$ such that either $\left|x\left(t_{1}\right)\right|=\varepsilon,\left|x\left(t_{2}\right)\right|=\varepsilon / 2$ and $\varepsilon / 2 \leqq$ $|x(t)| \leqq \varepsilon$ for all $t_{1} \leqq t \leqq t_{2}$ or $\left|x\left(t_{1}\right)\right|=\varepsilon,\left|x\left(t_{2}\right)\right|=3 \varepsilon / 2$ and $\varepsilon \leqq|x(t)| \leqq$ $3 \varepsilon / 2$ for all $t_{1} \leqq t \leqq t_{2}$. In either case we proceed as in the proof of Theorem 3 to contradict $V \geqq 0$ and the conclusion follows.

The most significant contribution of this note is in the showing that condition (ii) of Theorem 2 need not be checked in order to guarantee asymptotic stability. Of lesser significance is that we have weakened the condition on the right-hand side of system (1) by asking that $f(t, x)$ be of Carathéodory type rather than continuous.

\section{REFERENCES}

1. H. A. Antosiewicz, A survey of Liapunov's second method, Contributions to the Theory of Nonlinear Oscillations, vol. 4, Ann. of Math. Studies, no. 41, Princeton Univ. Press, Princeton, N.J., 1958, pp. 141-166. MR 21 \#1432.

2. Fred Brauer, Some refinements of Liapunov's second method, Canad. J. Math. 17 (1965), 811-819. MR 31 \#3670.

3. E. A. Coddington and N. Levinson, Theory of ordinary differential equations, McGraw-Hill, New York, 1955. MR 16, 1022.

4. W. Hahn, Theory and application of Liapunov's direct method, Prentice-Hall, Englewood Cliffs, N.J., 1963. MR 26 \#5230.

5. Maratschkow (V. Maračkov), Über einem Liapounoffschen Satz, Bull. Soc. Phys.Mat. Kazan. (3) 12 (1940), 171-174. MR 7, 158.

6. J. L. Massera, On Liapunoff's conditions of stability, Ann. of Math. (2) 50 (1949), 705-721. MR 11, 721.

7. T. Yoshizawa, Stability theory by Liapunov's second method, Math. Soc. Japan, no. 9, Math. Soc. Japan. Tokyo, 1966. MR 34 \#7896.

Department of Mathematics, Memphis State University, Memphis, Tennessee 38111 